\title{
Le réalisme social et l'école de Chicago : rencontre de la littérature et de la sociologie dans la communication pragmatiste chez John Steinbeck et Herbert Blumer
}

\section{Jean-François Côté}

Numéro 26, 1996

La sociologie saisie par la littérature

URI : https://id.erudit.org/iderudit/1002345ar

DOI : https://doi.org/10.7202/1002345ar

Aller au sommaire du numéro

Éditeur(s)

Département de sociologie - Université du Québec à Montréal

ISSN

0831-1048 (imprimé)

1923-5771 (numérique)

Découvrir la revue

Citer cet article

Côté, J.-F. (1996). Le réalisme social et l'école de Chicago : rencontre de la littérature et de la sociologie dans la communication pragmatiste chez John Steinbeck et Herbert Blumer. Cahiers de recherche sociologique, (26), 115-137. https://doi.org/10.7202/1002345ar

\section{Résumé de l'article}

Dans le contexte américain des années trente, la rencontre entre la littérature et la sociologie peut être en partie évaluée dans une comparaison entre le réalisme social de John Steinbeck, exemplifié dans son roman The Grapes of Wrath (Les raisins de la colère) et les développements méthodologiques de l'interactionnisme symbolique de Herbert Blumer, qui veulent assurer une suite à la tradition des études empiriques de l'école de Chicago. Cette rencontre, qui pose une possibilité de saisir l'expression sociale dans son contexte " naturel » et qui met en oeuvre une méthodologie "naturaliste » en sociologie faisant appel plus largement à des concepts hérités de la philosophie du pragmatisme, est en fait portée par l'idée d'une possibilité de communication " immédiate " de la vie sociale. On peut prendre la mesure critique de ce qui s'exprime alors dans ce contexte, en fonction des exigences de la réflexion actuelle en sociologie concernant les modalités d'analyse et d'expression du " monde vécu ", ainsi que des liens possibles à établir dans cette perspective entre littérature et sociologie. 


\section{Le réalisme social et l'école de Chicago: rencontre de la littérature et de la sociologie dans la communication pragmatiste chez John Steinbeck et Herbert Blumer}

Jean-François CÔTÉ

Le romancier John Steinbeck recevait, en 1940 aux États-Unis, le Social Work Today Award. La même année, et pour le même roman, The Grapes of Wrath (Les raisins de la colère), il recevait également le Pulitzer Prize et l'American Booksellers Association Award. Ces deux dernières distinctions, à proprement parler littéraires, bien qu'assez différentes dans leurs significations respectives, représentent en quelque sorte des étapes dans ce qui allait mener Steinbeck au prix Nobel de littérature, en $1962^{1}$. La première distinction est toutefois nettement plus intrigante, dans la mesure où elle touche un domaine (le «travail social») qui n'a de prime abord rien à voir avec la littérature ${ }^{2}$. Mais dans le contexte de l'entre-deux-guerres aux États-Unis, durant cette période de crise qui allait mener à une transformation profonde des structures de la société, à la faveur en particulier du New Deal de F. D. Roosevelt, critique sociale et réformisme pouvaient apparemment trouver des échos significatifs dans toutes les formes de l'expression culturelle; dans le monde politique comme dans le monde économique, dans la littérature comme dans les sciences sociales - la sociologie notamment.

1 Voir à ce sujet la chronologie dans John Steinbeck, The Grapes of Wrath. Text and Criticism, édition préparée par P. Lisca, New York, Penguin Books, 1972, p. xi-xiii. Le roman The Grapes of Wrath fut originalement publié en 1939. Je suppose connu ici ce classique de la littérature américaine, qui met en scène l'épopée de la famille Joad de l'Oklahoma jusqu'à la Californie, «portrait» si l'on veut saisissant de travailleurs agricoles en peine de trouver du boulot au cœur de la Dépression.

2 La revue Social Work Today fut en son temps, c'est-à-dire dans les années trente et jusqu'à son dernier numéro en 1942, une revue professionnelle engagée - et même par moments «radicale» - destinée aux travailleurs sociaux aux États-Unis. Voir à ce sujet J. H. Ehrenreich, The Altruistic Imagination, Ithaca, Cornell U.P., 1985, p. 102-138. Je ne possède pas d'information quant aux autres «prix» qu'elle décerna. 
Dans la mesure en effet où la tradition sociologique de l'école de Chicago se constituait depuis les années vingt en accord avec des visées d'études des mœurs réalisées «sur le terrain», cherchant à coller au plus près à la «réalité des choses» et prétendant parvenir ainsi à avoir une incidence directe sur certaines réformes sociales, sinon même à agir directement sur certaines situations, à l'image du travail social, on peut comprendre comment un roman comme celui de Steinbeck pouvait trouver une place pour ainsi dire privilégiée. On peut suggérer en outre que The Grapes of Wrath était porté par ce courant de réformes plus que par le mouvement des intellectuels et des écrivains «radicaux» engagés dans l'entreprise de redéfinition des paramètres culturels aux États-Unis d'alors ${ }^{3}$. Mais surtout, c'est dans l'esprit général de la philosophie du pragmatisme que l'on peut ranger bon nombre de ces diverses expressions, en montrant comment le réalisme social, qui avait fini par dominer la création littéraire de l'époque, reprenait sur le plan esthétique (ou plus précisément, poïétique) les prémisses de ce qui s'exprimait ailleurs, en philosophie ou en sociologie, ou même sur le plan «concret» d'un engagement immédiat dans l'action sociale, à travers la communication pragmatiste perçue comme seule issue de la signification - du point de vue tant de la littérature que de la sociologie ou du travail social ${ }^{4}$. Les sciences (sociales) à ce moment étaient tenues elles aussi de trouver le sens de leur propre engagement dans l'action sociale ${ }^{5}$; on peut alors penser que le Social Work Today Award aurait pu réellement être convoité par une partie de la sociologie engagée justement dans l'effort de réforme des structures sociétales. C'est en tout cas ce que suggère ce contexte général qui permettait une telle confusion des genres.

La réception du roman de Steinbeck à l'époque témoigne d'ailleurs éloquemment de cette confusion - qui reste malgré tout, à mon sens, étonnante - entre sciences sociales, littérature et travail social, domaines auxquels on peut aussi ajouter le journalisme de reportage et l'activisme

3 Et je ne parle pas tant ici du courant de la «littérature prolétarienne», qui proliféra sous les encouragements des partisans du Parti communiste américain et de la revue $\mathrm{New}$ Masses, jusqu'au milieu des années trente, que du courant du modernisme littéraire de la tradition américaine, celui d'une Gertrude Stein ou d'un John Dos Passos, ou même celui de William Faulkner.

4 Sur les rapports entre la sociologie de l'école de Chicago et la littérature, voir entre autres E. Rochberg-Halton, «Life, literature, and sociology in turn-of-the-century Chicago», dans S. J. Browner (dir.), Consuming Visions. Accumulation and Display of Goods in America, 1880-1920, New York, W.W. Norton and Co., 1989, p. 311-325.

5 Voir à ce sujet R. S. Lynd, Knowledge for What?, New York, Grove Press Inc., 1964 (1939). 
politique ${ }^{6}$; ainsi, dans un article paru en novembre 1939 dans la revue Forum, F. J. Taylor s'était cru obligé de «corriger les faits», en montrant comment une enquête personnelle sur ce même terrain de la migration de l'Oklahoma à la Californie lui permettait de réfuter ce que la fiction romanesque avait «déformé» - ce qui n'était en somme que bien peu en comparaison des réactions beaucoup plus vives soulevées en Oklahoma (comme en Californie) au regard des «descriptions» de ces migrant workers, de leurs conditions de vie et de travail, produites par Steinbeck, réactions rapportées notamment par des critiques tant littéraires que «sociales»; dénoncé par l'Associated Farmers of Kern County (Californie) pour sa «propagande communiste», le roman avait été banni par le Kansas City Board of Education, et quelques exemplaires furent même brûlés sur la recommandation du Library Board of East St. Louis?

Dans un ordre d'idée parallèle, qui montre la portée tout à fait réaliste - sinon purement polémique! - du roman de Steinbeck, un article de la revue Fortune avait, à peu près simultanément à sa parution en 1939, développé une interrogation sur la situation misérable de ces travailleurs migrant vers la Californie, en fuite devant la dévastation du dust bowl du Midwest américain. Par ailleurs, une commission d'enquête gouvernementale, sous la direction de la Work Progress Administration (WPA), avait également été formée dans le cadre des

6 Peter Lisca écrit: «When The Grapes of Wrath was published in April of 1939, there was little likelihood of its being accepted and evaluated as a piece of fiction. Because of its nominal subject, it was too readily confused with such high-class reporting as Ruth McKenny's Industrial Valley, the WPA collection of case histories called These Are Our Lives, and Dorothea Lange and Paul S. Taylor's An American Exodus. The merits of The Grapes of Wrath were debated as social documentation rather than fiction.» (P. Lisca, «The Grapes of Wrath as fiction», dans J. Steinbeck, ouvr. cité, p. 729.) Il faut voir la coïncidence de la publication du roman avec les débuts de la tradition du «reportage photographique», tel qu'il se développe avec la revue Life fondée en 1936, et avec d'autres ouvrages de photo-documentation célèbres de l'époque, tels celui de J. Agee et W. Evans, Let Us Now Praise Famous Men, ou de E. Caldwell et M. Bourke-White, You Have Seen their Faces. Les médias en général sont touchés à ce moment par une préoccupation renouvelée de «coller» à la réalité sociale, comme le montre bien M. Schudson, Discovering the News, New York, Basic Books, 1978. L'activisme politique qu'on trouve dans le domaine syndical ou dans le domaine social est à l'image des préoccupations de la période, comme le révèlent les témoignages et les analyses et de R. H. Pells, Radical Visions and American Dream, Middletown (Conn.), Wesleyan University Press, 1984 (1973), et W. Susman (dir.), Culture and Commitment, New York, George Braziller, 1973.

7 Voir F. J. Taylor, «California's Grapes of Wrath», C. McWilliams, «California pastoral», M. Shockley, "The reception of The Grapes of Wrath in Oklahoma». Tous ces articles sont repris dans J. Steinbeck, ouvr. cité, p. 643-691. 
programmes du New Deal pour tenter d'établir des normes de travail unifiées dans différents secteurs de l'activité économique pour trouver des solutions à ce problème social ${ }^{8}$. La mobilisation générale autour de cette question en faisait en quelque sorte un emblème de la situation américaine, au temps de la Dépression. Mais elle soulignait aussi des enjeux dont nous sommes encore aujourd'hui pour l'essentiel tributaires.

En effet, la situation décrite sommairement ici ne possède pas qu'un caractère anecdotique, ou encore une portée historique à mettre au compte des curiosités culturelles ou intellectuelles du XXe siècle; si l'on $\mathrm{y}$ regarde de plus près, on y retrouve même en fait un nœud de questions qui rejaillissent à l'heure actuelle, pour la sociologie, avec une ampleur surprenante ${ }^{9}$. Ce que semble «résoudre» pour ainsi dire le roman de Steinbeck, c'est le rapport entre la réalité et sa représentation, d'un côté, la situation d'une expérience de vie misérable qui exige des changements dans le cadre d'un ordre politico-économique déshumanisé, de l'autre, et enfin la conciliation d'une certaine philosophie sociale avec l'engagement (individuel) permettant la transformation de ces conditions de vie (générales) inadéquates. Dans les mots de Steinbeck, son propre engagement littéraire du temps allait de pair avec l'admiration qu'il portait à ces gens ordinaires, convaincu que «[...] out

8 Fortune, «I wonder where we can go now», dans J. Steinbeck, ouvr. cité, p. 623-642. Dans la foulée des programmes mis sur pied comme mesures temporaires d'emplois, la WPA avait lancé un programme s'adressant à des écrivains, qui avaient pour tâche de préparer des «guides» touristiques portant sur chacun des États. Ce qu'il y a d'intéressant à ce sujet est le traitement réservé à la question des migrant workers dans le guide de la Californie; ceux-ci sont assimilés à des travailleurs en manque d'organisation (syndicale). Voir Federal Writers' Project, California, New York, Hastings House, 1939 , p. 106.

9 C'est dans le cadre des études en méthodologie «qualitative» que ce débat se déploie, en particulier autour de la tradition de l'interactionnisme symbolique. Voir à ce sujet K. Plummer, «Herbert Blumer and the life history tradition», Symbolic Interaction, vol. 13, no 2, 1990, p. 125-144; N. K. Denzin, «The spaces of postmodernism: Reading Plumer on Blumer», Symbolic Interaction, vol. 13, no 2, 1990, p. 145-154; K. Plumer, «Staying in the empirical world: Symbolic interactionism and postmodernism. A response to Denzin», Symbolic Interaction, vol. 13, no 2, 1990, p. 155-160. Mais ce débat ne se limite évidemment pas à la sociologie américaine; l'ouvrage récent publié par P. Bourdieu (dir.), La misère du monde, Paris, Seuil, 1993, se situe très exactement dans une visée analytique similaire de prise en considération de la réalité vécue et de participation à la transformation éventuelle des situations mises en cause - visée que l'on rencontre également dans le cadre plus général du «retour de l'acteur» et de l' «analytique participative» des mouvements sociaux dont Alain Touraine est le représentant éminent. Sur ces questions, voir J. Hamel, «Quelques problèmes de la méthodologie qualitative en sociologie», Revue européenne des sciences sociales, t. 32 , no 98,1994 , p. 45-61. 
of those qualities will grow a new system and a new life which will be

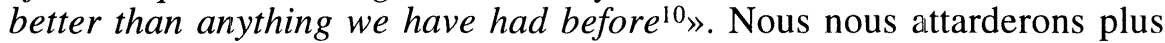
bas à la façon par laquelle Steinbeck avait, en son temps, "résolu» les questions énumérées ci-dessus. Mais pour les reformuler dans un vocabulaire qui fera apparaitre toute leur actualité, on peut suggérer que trois ordres de questions concernent ici directement la sociologie contemporaine: 1) le rapport conflictuel entre «système» et «monde vécu», pour employer la formulation habermassienne du problème qui semble même évoquée par Steinbeck dans la citation précédente, au moment où il formule le souhait d'une adéquation plus juste entre les deux «niveaux» d'existence de la vie sociale; 2) l'adéquation des représentations (ou des concepts) avec la réalité qu'ils sont censés «comprendre»; 3) le rapport qu'entretient la sociologie avec la société, par le biais de l'exigence d'une connaissance scientifique de la réalité. Je vais développer brièvement ces trois ordres de questionnement, à la lumière de certains débats actuels en sociologie, en tentant de montrer également en quoi l'exemple du réalisme social de Steinbeck et sa possible confusion (ou du moins sa proximité) avec le pragmatisme de l'école de Chicago continue de nous instruire sur le rapport entre sociologie et littérature, et peut-être surtout sur l'importance d'une distinction des fonctions symboliques imparties à chaque domaine d'activité.

\section{L'opposition entre «système» et «monde vécu»}

La question de l'opposition entre «système» et «monde vécu» travaille la sociologie contemporaine d'une manière qui met en jeu non seulement ce problème en soi, constitué comme on le sait par l'évolution autonome des différentes sphères d'activité - et particulièrement l'économie et la technologie dans leur dimension de «rationalisation effective et cumulative des opérations de la pratique» - par rapport à la vie sociale globale, mais encore la «méthode» sociologique elle-même. Elle touche la façon dont la sociologie prétend parvenir à l'objectivation des phénomènes de la réalité humaine, en vertu d'une «abstraction» qui fait par moments verser son autonomie théorique dans un espace totalement détaché de la «réalité sociale». Le systémisme, dans ce sens, apparaît en sociologie comme la formulation théorique qui, par un certain retournement ironique, en arrive à parfaire la vision d'un processus à l'œuvre objectivement dans la société en en donnant la

10 «Steinbeck's suggestion for an interview with Joseph Henry Jackson», dans J. Steinbeck, ouvr. cité, p. 862. Je souligne. 
cohérence et la justification, sinon la légitimité, «scientifiques ${ }^{11}$ ». Jürgen Habermas s'est attaqué depuis un certain temps déjà à ce problème, en proposant d'opposer aux visées systémiques d'abord une critique théorique du systémisme lui-même, puis une mise en relief des conditions par lesquelles le «monde vécu» peut accéder à une représentation plus juste dans l'ordre des choses contemporain ${ }^{12}$. La solution à ce problème proposée par Habermas reste toutefois éminemment paradoxale, puisque tout en voulant mettre de l'avant la dimension «expressive» immanente au monde vécu, et en voulant privilégier pour ce faire une approche basée sur une appréhension esthétique de la vie sociale, son programme théorique s'est jusqu'à un certain point enfermé dans le formalisme propre à la pragmatique des actes de langage, transposé dans les termes de l' «agir communicationnel ${ }^{13^{3}}$. Dans un sens, la reconnaissance des «conditions formelles» d'établissement du dialogue «sans contrainte» que vise Habermas deviendrait l'enjeu de l'analyse, et ce plus, à la limite, que le «contenu» (la signification) véritable de ce qui s'exprime. En dépit des réserves que j'émettrais à l'égard de la formulation même du problème de l'expression du «monde vécu» dans la théorie habermassienne, on peut opposer le fait que d'autres approches ont, depuis longtemps, fait montre dans ce sens d'une plus grande efficacité (et peut-être aussi

11 Et l' «ironie», peut-on ajouter à cet égard, est simplement le fait d'une production scientifique qui, par souci de positivité, ignore les conditions de sa propre production en tant que connaissance, discours, etc. C'est donc la position «objectiviste» de la description systémique qui produit ce positivisme; le problème n'est pas alors de remettre en question l'objectivité des descriptions, mais plutôt de s'interroger sur la «genèse» sociale et historique de cette objectivité, en gardant à l'esprit que l'expression scientifique, loin d'être «neutre» dans ce procès, représente une contribution à l'édifice culturel qu'elle érige (à l'endroit de la nature autant que de la société). Il faut faire remarquer cependant ici que l' «abstraction systémique» est celle qui «colle» de plus près aux développements de la société contemporaine - et donc celle qui participe le plus activement à l'expression de ce mode de développement sociétal.

12 Voir J. Habermas, notamment «Progrès technique et monde vécu social», $L a$ technique et la science comme idéologie, trad. par J. R. Ladmiral, Paris, Denoël/Gonthier, 1973, p. 75-96, de même que «La colonisation du quotidien», trad. par G. Raulet, Esprit, no 36, décembre 1979, p. 34-51, et The Theory of Communicative Action, vol. 2: Lifeworld and System: A Critique of Functionalist Reason, trad. par T. McCarthy, Boston, Beacon Press, 1989.

13 C'est en fait depuis son ouvrage Communication and the Evolution of Society, trad. par T. McCarthy, Boston, Beacon Press, 1979, qu'Habermas s'est résolument engagé dans la perspective d'une solution "communicationnelle» aux problèmes d'analyse théorique (et de solution pratique) de la société contemporaine. On trouve une critique, développée sur une base «néopragmatiste» de la théorie de l'agir communicationnel d'Habermas chez E. Rochberg-Halton, «Jürgen Habermas's theory of communicative etherealization», Symbolic Interaction, vol. 12, no 2, 1989, p. 333-353. Je reviendrai dans la toute dernière partie de ce texte sur cette position néopragmatiste. 
jusqu'à un certain point d'une plus grande naïveté...), en proposant tout simplement de «présenter» l'expression brute du monde vécu dans les termes où elle apparaît, s'effaçant pour ainsi dire derrière leur objet pour lui donner la parole. Ce qu'on a appelé le domaine de la méthodologie qualitative $a$, en particulier à partir des années vingt et trente aux États-Unis, élaboré une quantité impressionnante d'approches visant cet objectif. De l'étude classique et fondatrice de W. I. Thomas et F. Znaniecki sur The Polish Peasant, en passant par l'interactionnisme symbolique de Herbert Blumer, jusqu'aux avancées récentes de l'ethnométhodologie, on peut en effet considérer que la «présentation du monde vécu» est apparue comme un domaine privilégié de l'expression sociologique contemporaine, domaine visant plus ou moins expressément les «inconséquences» de l'approche systémique ${ }^{14}$. Le problème devient évidemment par la suite, pour ce type d'approches, de parvenir à définir des concepts qui puissent apparaître pleinement comme des «représentants» légitimes des situations à l'étude, ou comme des «avancées» significatives dans le domaine de la connaissance.

Nous reviendrons en détail sur ce problème de la définition du concept ainsi que sur la définition de son «rôle» dans la vie sociale; pour le moment, je voudrais simplement suggérer qu'il y a en effet un certain rapprochement à établir entre l'expression sociologique du monde vécu et l'entreprise littéraire elle-même, et même un rapprochement double. D'une part, dans la mesure où le réalisme littéraire cherche par exemple, comme chez Steinbeck, à déployer une forme esthétique qui va donner l'illusion de faire transparaître la "vraie vie» dans le roman, en dégageant de manière convaincante un «effet de réel», la représentation cherchera à coller au plus près de ce qui fait l'expression même de ce monde vécu: ainsi, les dialogues dans The Grapes of Wrath sont des dialogues «parlés»; ils «rendent» ou imitent le langage vernaculaire tel qu'on l'entend réellement de la bouche de ces travailleurs agricoles, et donc la forme littéraire correspond ici à la vie sociale dans la mesure où elle se plie (sur les plans phonétique, syntaxique, sémantique, etc.) à ce qu'elle veut représenter. Bien sûr, les dialogues ne sont pas le seul élément de la narrativité en jeu; les descriptions, les personnages, la «mise en intrigue» constituent autant de problèmes redoutables qu'affronte la création littéraire du réalisme social de l'époque, et les solutions qu'elle apporte, en empruntant

14 Je ne distingue pas pour le moment ces approches qui sont, en tout ou en partie, héritières des développements de l'école de Chicago et surtout de ses fondements ontoépistémologiques dans le pragmatisme américain. Je vais toutefois réserver l'essentiel de mes remarques ultérieures aux positions de l'interactionnisme symbolique, qui assume ces fondements dans le développement de sa propre méthodologie de recherche. 
notamment au modernisme littéraire quelques-unes de ses «techniques», constituent autant de solutions au problème global de la représentation $^{15}$. D'autre part, mais ici le rapprochement est relativement trompeur, la distance esthétique qui est nécessairement prise par l'entreprise littéraire se compare à la distance théorique tout aussi nécessairement prise par l'entreprise sociologique ${ }^{16}$; toutes deux se constituent par rapport à l'expression première du monde vécu, sur un mode «décalé» dans le temps et l'espace par rapport à elle. Mais leur proximité est relativement trompeuse dans ce cas, je le répète, puisque l'entreprise sociologique ne poursuit pas les mêmes fins que l'entreprise littéraire. Les deux «distances» en cause donnent lieu à des espaces d'expression différents - que l'on pourrait toutefois éventuellement confondre, comme en témoigne l'exemple qui nous intéresse $^{17}$. Dans cette perspective, le travail de l'imagination, l'invention et la «fiction», ainsi que le plaisir esthétique, sont des dimensions de la création littéraire que l'analyse sociologique ne vise pas au premier chef. Mais peut-on être si sûr qu'il n'y a pas de plaisir esthétique dans l'activité de connaissance ${ }^{18}$ et dans l'élaboration de la représentation de la réalité auxquelles se livre la sociologie en tant que science? Et qu'il n'y a pas une part d'invention et de «fiction» dans l'activité scienti-

15 Sur la question des rapports entre le réalisme social de Steinbeck et le modernisme, qui pourrait après ce que l'on a dit sembler tout à fait étrangers l'un à l'autre, voir C. E. Magny, L'âge du roman américain, Paris, Seuil, 1948, et P. Lisca, art. cit., p. 729747. Steinbeck utilise notamment, dans des chapitres intercalés dans des chapitres qui développent l'intrigue du roman, la description presque «photographique» de lieux, de routes ou de bordures de routes qui tente de donner une «image» aussi «fidèle» que possible de la réalité des détails du trajet de ses personnages.

16 Par «distance théorique», j'entends autant celle qui opère par le biais de la simple description que celle qui paraît s'effacer devant la «présentation» de la réalité sociale. Je reviendrai sur ce point dans la prochaine section.

17 Je souligne de nouveau que cette question du rapprochement (ou même de la fusion!) entre création littéraire et analyse sociologique est tout à fait d'actualité, comme en témoignent des ouvrages récents tels celui de J. Van Maanen, Tales of the Field, Chicago, University of Chicago Press, 1988, ou encore celui de J.-F. Laé et N. Murard, Les récits du malheur, Paris, Descartes et cie, 1995.

18 Il s'agit évidemment d'un problème qui, comme on le sait, est assez ancien, puisqu'il se manifeste au tout début de la Métaphysique d'Aristote, qui lie explicitement l'activité théorique au plaisir (et en particulier au plaisir de la vision - formulation qui anticipe la réflexion moderne sur la perception). On peut souligner également que cette dimension de «plaisir» de l'activité de connaissance peut être interprétée dans une perspective psychanalytique, qui envisage par exemple le rôle que joue le refoulement du désir subjectif individuel puis sa «libération» dans la contrainte de l'écriture et de la représentation réaliste témoignant de son rôle d'autorité et de pouvoir, notamment dans l'écriture ethnographique, comme le fait dans une optique de critique féministe et poststructuraliste P. T. Clough, The End(s) of Ethnography. From Realism to Social Criticism, Newbury Park (Calif.), Sage Publications, 1992. 
fique? Inversement, est-on si sûr que la création esthétique est libre de tout «concept» ou de toute attache conceptuelle dans son travail de la forme? Je propose, pour répondre à ces questions, d'interroger plus à fond le rapport entre le concept et la réalité tel qu'il se développe dans les approches d'analyse qualitative du monde vécu, en montrant en parallèle, mais pour ainsi dire à rebours, quel rôle joue le concept dans la création esthétique du réalisme social de Steinbeck.

\section{L'adéquation du concept et de la réalité}

La question épistémologique de l'adéquation du concept et de la réalité, telle qu'elle peut se poser dans le cadre de la représentation du monde vécu, semblerait selon toute vraisemblance renvoyer au débat typiquement moderne qui a opposé rationalisme et empirisme pendant l'épanouissement de la modernité (opposant une tradition de pensée depuis Bacon jusqu'à Locke et Hume à une autre, par exemple de Descartes à Kant). C'est ce débat qui pourrait en effet sembler être repris, mutatis mutandis, dans le cadre de l'opposition entre système et monde vécu. Or il n'en est rien. Depuis Hegel, et plus largement dans la perspective de constitution d'une phénoménologie véritable, ce débat entre rationalisme et empirisme s'est en quelque sorte fondu à un tout autre débat, qui oppose davantage une science objective et une science réflexive - c'est-à-dire une science qui produit positivement une objectivation des phénomènes et une autre qui reconnaît réflexivement son rôle dans ce travail d'objectivation, deux orientations qu'on a pris l'habitude de définir respectivement comme étant celle des «sciences de la nature» et celle des «sciences de l'esprit» (Geisteswissenschaften), ces dernières qu'on a aussi parfois aussi appelées «sciences de la culture». Particulièrement mis en évidence dans les sciences humaines ${ }^{19}$, les paramètres de ce débat acceptent que la réalité soit en fait comprise à la fois dans sa dimension sensible («matérielle») et sa dimension idéelle («spirituelle») - bien que, ici, ce soit justement la question de savoir quelle dimension prime l'autre dans le développement de la réalité qui s'impose, et conséquemment quelle place la connaissance s'y reconnaît. C'est dire que la définition de l'essence et de l'existence humaines et

19 Mais pas seulement pour elles, puisque les sciences naturelles posent (ou plutôt: supposent) elles aussi, mais sans le problématiser, la question du sujet de la connaissance. Sur cette question, voir M. Freitag, «De l'objectivité donnée à sa genèse sociale», Épistémologie sociologique, nos 15-16, 1973, p. 43-62. Sur la façon dont on reconnaît la participation active de l'activité scientifique dans l'objectivation des phénomènes, plus particulièrement dans le procès d'écriture qu'elle met en œuvre, voir P. Ouellet, Voir et savoir. La perception des univers du discours, Candiac, Les Éditions Balzac, 1992. 
leur saisie par l'expérience de la connaissance n'ont cessé de se poser dans leur rapport intrinsèque depuis lors; ainsi, pour la sociologie classique, de Comte à Marx, ou de Spencer à Weber et jusqu'à Durkheim, ce débat ne s'est-il jamais définitivement résolu, bien qu'il apparût toujours en toile de fond de leurs analyses respectives. Mais il se déploie du fait qu'il se tient justement dans l'horizon d'une définition ontologique de l'expérience qui comprend toujours des propositions rejoignant la perception de l'existence humaine. Le pragmatisme, qui est comme on le sait à l'origine des méthodes d'analyse du monde vécu aux États-Unis, n'est pas non plus, à son corps défendant, exempt de propositions quant à l'essence de l'existence humaine, et cela même lorsqu'il prétend ne prêter attention qu'à cette dernière dans ses analyses «empiriques» de la réalité sociale. Il y a toujours en d'autres termes une théorie implicite (sinon une «vision du monde») dans l'entreprise de présentation du monde vécu; c'est dire qu'existe pour elle un «fondement» ontologique revendiqué au moins implicitement, ou du moins assumé - et cela jusque dans les positions extrêmes qui maintiennent expressément qu'il n'y a «pas de fondement», car c'est cette proposition même, avec tout ce qu'elle masque au chapitre des présupposés («naturalistes» par exemple), qui devient alors justement fondamentale. Sans insister ici sur la question complexe de l'ontologie du pragmatisme (qui entraîne des développements du côté proprement métaphysique de la question), on peut tout simplement mentionner que nous sommes ici en présence d'une ontologie à caractère naturaliste ${ }^{20}$. Cette définition de l'expérience humaine dans des termes naturalistes a évidemment une incidence directe sur la perception de l'existence humaine ainsi que sur la conception de l'expérience de la connaissance et la mise en œuvre d'une méthodologie conséquente, puisqu'elle définit, dans l'interactionnisme symbolique d'un Herbert Blumer, par exemple, toute la légitimité d'un projet de connaissance naturaliste de la vie sociale, c'est-à-dire un projet centré sur l'étude empirique de la signification dans la perspective des sciences naturelles ${ }^{21}$.

20 Voir J. Dewey, «Experience and nature», dans J. A. Boydston (dir.), The Later Works, vol. 1, Carbondale and Edwardsville, Southern Illinois University Press, 1981 (1925).

21 Blumer prend appui, dans son orientation «naturaliste», sur une méthodologie empirique qui aurait selon lui ses sources chez Darwin. Il écrit: «Exploration and inspection, representing respectively depiction and analysis, constitute the necessary procedure in direct examination of the empirical social world. They comprise what is sometimes spoken of as "naturalistic" investigation-investigation that is directed to a given empirical world in its natural, ongoing character instead of a stimulation of such a world, or to an abstraction from it (as in the case of laboratory experimentation), or to a substitute for the world in the form of a preset image of it. The merit of naturalistic study is that it respects and stays close to the empirical domain. This respect and 
On pourrait montrer que cette position de l'interactionnisme symbolique est contradictoire. Car tout en reconnaissant que le concept joue, pour les sciences en général, un rôle constitutif dans ce qui les fait être ce qu'elles sont - c'est-à-dire un ordre de perception comportant des dimensions conceptuelle et praxéologique spécifiques ${ }^{22}-$, Blumer considère que c'est toujours l'épreuve de la réalité empirique qui est décisive pour l'entreprise scientifique ${ }^{23}$. Mais cette contradiction apparente n'est en somme qu'un malentendu. Car la «réalité» se

closeness is particularly important in the social sciences because of the formation of different worlds and spheres of life by human beings in their group existence. [...] Naturalistic inquiry, embracing the dual procedures of exploration and inspection, is clearly necessary in the scientific study of human group life. It qualifies as being "scientific" in the best meaning of that term.» (H. Blumer, Symbolic Interactionism. Perspective and Method, Berkeley, University of California Press, 1986 (1969), p. 4647.) En dehors (ou en deçà) de ses préoccupations d'ordre méthodologique, Blumer reconnaît aussi explicitement son affiliation au pragmatisme de G. H. Mead et de J. Dewey (lorsqu'il écrit par exemple: «The term "symbolic interactionism" has come into use as a label for a relatively distinctive approach to the study of human group life and human conduct. The scholars who have used the approach or contributed to its intellectual fondation are many, and include such notable figures as George Herbert Mead, John Dewey, W. I. Thomas, Robert E. Park, William James, Charles Horton Cooley, Florian Znaniecki, James Mark Baldwin, Robert Redfield, and Louis Wirth.» (1969); voir également à ce sujet C. W. Tucker, «Herbert Blumer: A pilgrimage with pragmatism», Symbolic Interactionism, vol. 11, no 1, 1988, p. 99124.

22 Blumer écrit à ce sujet: «The scientific concept, as a way of conceiving, enables one to circumvent problems of perceptual experience; the content of the scientific concept consists of an abstracted relation which becomes the subject of separate and intensive study; the concept, because of its verbal character, may be shared, and so it permits concerted activity in scientific procedure; scientific concepts in their interrelation make possible the structure of science. [...] As I see it, the concept more specifically considered serves three functions: (1) it introduces a new orientation or point of view; (2) it serves as a tool, or as means of transacting business with one's environment; (3) it makes possible deductive reasoning and so the anticipation of new experience.» (H. Blumer, Symbolic Interactionism, ouvr. cité, p. 163.)

23 Il écrit: "Reality" for empirical science exists only in the empirical world, can be sought only there, and can be verified only there. [...] One errs if he thinks that since the empirical world can exist for human beings only in terms of images or conceptions of it, therefore reality must be sought in images or conceptions independent of an empirical world. Such a solipsistic position is untenable and would make empirical science impossible. The position is untenable because of the fact that the empirical world can "talk back" to our pictures of it or assertions about it-talk back in the the sense of challenging and resisting, or not bending to, our images or conceptions of it. This resistance gives the empirical world an obdurate character that is the mark of reality. [...] Fundamentally, empirical sciences is an enterprise that seeks to develop images and conceptions that can successfully handle and accomodate the resistance offererd by the empirical world under study.» (Ibid., p. 22-23.) 
compose précisément de ces deux dimensions, «matérielles» et «idéelles ${ }^{24}$, et l'expérience de la connaissance n'est jamais comprise que dans le rapport qui unit ces deux dimensions, ou en d'autres mots, et plus exactement, par la tension de ce rapport qui confirme ou infirme leur union. Comme l'a bien démontré à ce sujet Ernst Cassirer, le problème de la signification dans l'expérience humaine, du point de vue de la théorie de la connaissance, n'est pas de statuer à chaque fois sur la «vérité objective» et la «validité» d'un aspect particulier ou de l'ensemble de l'expérience, mais bien de savoir «si tel ou tel contenu, livré par l'expérience, occupe une place durable ou transitoire au sein de cet ensemble ${ }^{25}$ ». Bref, il n'existe pas de matérialité empirique «en soi», sans rapport avec la dimension spirituelle de l'existence, pas plus qu'il n'existe d'idéal transcendantal «en soi», sans rapport avec la dimension sensible de l'existence; c'est bien plutôt le rapport entre ces termes qui définit fondamentalement ce que nous considérons en général comme étant la réalité, cette dernière se composant toujours ainsi, par exemple, du lien entre faits et valeurs. La connaissance scientifique intervient dès lors comme une réflexion de second degré (comme une «critique réflexive» pourrait-on dire), au regard de la façon dont ce rapport se définit, en l'insérant dans un ensemble réflexif plus large, compris comme nouveau rapport structurant la réalité. Estce à dire pour autant qu'il n'y a pas dans ce contexte de différence entre les sciences de la nature et les sciences de la culture (ou les sciences de l'esprit)?

Ce serait plutôt l'inverse, car en fait, une fois admis que c'est le rapport entre les dimensions sensibles et spirituelles de l'existence humaine qui est constitutif de la réalité, toute la question porte

24 Ce que reconnaît par ailleurs Blumer lui-même, dans l'énumération sommaire des catégories d' «objets» qui composent la vie sociale à laquelle il se livre (ibid., p. 1011). Mais à ce titre déjà subsiste une certaine ambiguïté, puisque tout en insistant sur le fait que c'est toujours la signification qui est en jeu dans le rapport entre les individus et les objets, on a parfois l'impression que ces objets possèdent une dimension empirique en soi.

25 E. Cassirer, «Le concept de réalité», Substance et fonction. Éléments pour une théorie du concept, trad. par P. Caussat, Paris, Minuit, 1977, p. 315. Cassirer ajoute sur ce point (p. 317): «C'est donc bien la différenciation logique des contenus d'expérience et leur insertion ordonnée dans un système de dépendances articulées qui forment le noyau véritable du concept de réalité. Articulation qui se trouve confirmée, s'il en est besoin, pour peu que l'on examine de près le fondement logique de l'expérimentation scientifique, porte-parole par excellence de la réalité empirique. Loin de se réduire à un simple compte rendu des faits perceptifs donnés hic et nunc, l'expérimentation scientifique n'a de valeur que dans la mesure où elle soumet les données particulières à un point de vue déterminé à partir d'une visée judicatoire. Ce faisant, elle leur confère une signification qu'elles ne possèdent pas dans le simple vécu sensible.» 
précisément sur la distinction des domaines de connaissance, qui se constituent dans des horizons fonctionnels différenciés. Et l'on peut montrer que, pour l'interactionnisme symbolique, tout le développement «méthodologique» se faisant dans la direction des sciences de la nature n'a en fait qu'un seul objectif: celui de se fondre positivement à cette réalité, ce qui lui assure un caractère de scientificité ${ }^{26}$. On peut soutenir que cette position constitue tout aussi bien une contribution de la connaissance scientifique non pas tant à la connaissance de la réalité qu'à sa transformation. Le «concept» ici possède essentiellement une fonction instrumentale, c'est-à-dire qu'il n'a pour but que de s'appliquer ou de se «résoudre» dans la situation à l'étude, comme il «résout» la signification de cette dernière ${ }^{27}$. Et c'est dans ce procès d'effacement de la distance que se comprend le projet positif des sciences de la nature, alors que le projet réflexif des sciences de la culture se comprend plutôt dans un autre sens, et presque à l'inverse, comme la reconnaissance d'une production de la distance, ce qui est le propre de l'expression symbolique comprise comme médiation - sans pour autant que cette distance soit comprise comme une pure

26 Blumer écrit à ce sujet: «Symbolic interactionism is a down-to-earth approach to the scientific study of human group life and human conduct. Its empirical world is the natural world of such group life and conduct. [...] Its methodological stance, accordingly, is that of direct examination of the empirical social world [...]. Symbolic interactionism recognizes that the genuine mark of an empirical science is to respect the nature of its empirical world - to fit its problem, its guiding conceptions, its procedures of inquiry, its techniques of study, its concepts, and its theories to that world. [...] Certainly, the basic premises of symbolic interactionism [...] have to have their empirical validity tested. If they cannot survive that test, they, together with the scheme of symbolic interactionism which they undergird, should be thrown ruthlessly aside. [...] The manner of testing the premises is to go to the empirical social world since the premises are declarations of the nature of that world.» $(\mathrm{H}$. Blumer, Symbolic Interactionism, ouvr. cité, p. 47, 48, 49.)

27 Blumer écrit ainsi: «Let us recognize the instrumental character of the concept inside the field of science. By accepting it in this character and using it critically perhaps we can avoid being mere bookkeepers of facts or spinners of metaphysics.» Il écrit également: «The success of the activity to which it gives rise becomes the test of the effectiveness of the concept. The concept is thus bounded on one side by frustrated activity and on the other by consequences which arise from the activity to which it gives direction. In so far as it lies between these portions of an act it has the character of a tool. At first, like all tools, it may be crude and may be used quite experimentally; later, like perfected tools, it may become refined and its use quite standardized.» (Ibid., p. 170, 166.) P. T. Clough («Herbert Blumer: A methodology for writing observation», ouvr. cité, p. 29-45) interprète cette position de Blumer en fonction d'une détermination «technologique» inspirée de la cinématique. Bien que cette analyse soit pénétrante en plusieurs points, il me semble plus opportun de situer le débat sur un autre plan, qui conserve l'enjeu d'une définition de l'épistémologie correspondant aux fondements ontologiques de l'expérience reconnus par le naturalisme en cause. 
«abstraction ${ }^{28}$ ». Dans le cas des sciences de la nature, cet effacement de la distance participe à mon avis de la reconnaissance seulement implicite de la responsabilité humaine investie non pas simplement dans la connaissance objective de la nature, mais dans sa transformation effective - puisque les sciences de la nature, en transformant notre perception des processus naturels, transforment leur «être», et s'ouvre alors explicitement un champ de manipulations de ces processus, dont héritent la technologie et la technique. Appliquée au domaine de la culture, on voit donc qu'une approche dérivée directement des sciences de la nature, qui abdique dans la reconnaissance explicite de sa propre responsabilité dans le procès d'objectivation des phénomènes de la réalité humaine produisant une «distance» (théorique) à leur égard, mène, et cela très paradoxalement, à la possibilité d'un social engineering ou d'une intervention immédiate totalement «extérieure» à la situation étudiée ${ }^{29}$. Nous sommes donc renvoyés sur ce plan au rapport qui s'établit entre les sciences sociales, ou la sociologie, et la société, et à l'évaluation de ce rapport dans le contexte la problématique de la méthodologie qualitative de l'école de Chicago et de ses suites actuelles. Nous reviendrons sur cette question dans la prochaine section.

Pour le moment, et si l'on retient ce qui vient d'être dit sur la distinction des approches des sciences de la nature et des sciences de la culture, il est aisé de voir comment le réalisme social à l'œuvre chez Steinbeck produit un «effet de réel» à même d'être comparé à celui qui est produit dans le cadre d'une appréhension naturaliste du monde. C'est par sa visée d'effacement de la distance dans l'expression, donnant une certaine impression de participation directe ou immédiate à la vie du groupe, que le roman de Steinbeck se rapproche des visées de la sociologie du monde vécu. Sur ce plan, il semble même que la poïétique de The Grapes of Wrath serait au plus près des visées de l'interactionnisme symbolique - si ce n'est que son entreprise est une œuvre de fiction, de création imaginaire, et non une entreprise d' «analyse empirique» de situation, le paradoxe étant donc cette fois que cette création «purement imaginaire» se rapproche le plus de ce

28 Cassirer précise cette idée en écrivant: «Par conséquent le langage n'est absolument pas une simple distanciation par rapport à nous-mêmes, mais au contraire, comme l'art et toute autre "forme symbolique", il nous ramène à nous-mêmes; il est créatif, en ce sens qu'à travers lui seulement se constitue la conscience de soi.» (E. Cassirer, Logique des sciences de la culture, trad. par J. Carro, Paris, Cerf, 1991, p. 137.)

29 Mais on peut comprendre du même coup pourquoi l'interactionnisme symbolique de Blumer multiplie les précautions pour contrecarrer une telle éventualité; c'est que tout son projet est en fait paradoxal, puisqu'en se développant dans un souci d'efficacité pratique son intervention ne peut que jouer dans la transformation immédiate de la vie sociale («naturelle») qui fait l'objet de l'analyse. 
qu'elle prétend représenter, alors que l'entreprise scientifique de l'interactionnisme symbolique de son côté veut pour ainsi dire «s'empêcher» de créer une représentation qui serait «trop distante» de son objet. Mais c'est en aval de cette dimension productive de l'activité (littéraire ou scientifique) que se tient la coïncidence réelle entre science («naturelle») et littérature; Steinbeck hérite en effet des principales conceptions - et sinon directement d'une position quant à la définition ontologique, du moins de la vision du monde - du naturalisme pragmatiste ${ }^{30}$. Pour Steinbeck, les formes de vie biologiques, et leurs exigences particulières, trouvent (ou devraient trouver...) un équivalent dans le domaine des formes culturelles de la vie sociale ${ }^{31}$. Cela a notamment comme conséquence que l'organisation du vivant possède à ses yeux une résonance dans la vision «organique» — ou même «organisationnelle» - de la culture. Dans cette perspective, les individus doivent être soumis à une «organisation sociale» effective, c'est-à-dire

30 Et cela fait du roman non pas une création «inductive», partant des faits du monde vécu pour les élever à leur représentation esthétique, mais bien au contraire une œuvre totalement «déductive» dans son propos, partant de concepts ou d'idées génériques pour en montrer les manifestations sensibles par la représentation esthétique. F. I. Carpenter écrivait d'ailleurs à ce sujet, en 1941: «Beside and beyond their function in the story, the ideas of John Steinbeck [...] possess a significance of their own. They continue, develop, integrate, and realize the thought of the great writers of American history. Here the mystical transcendantalism of Emerson reappears, and the earthly democracy of Whitman, and the pragmatic instrumentalism of William James and John Dewey. And these old philosophies grow and change in the book until they become new. They coalesce into an organic whole. And, finally, they find embodiment in character and action, so that they seem no longer ideas, but facts. The enduring greatness of The Grapes of Wrath consists in its imaginative realization of these old ideas in new and concrete forms. Jim Casy translates American philosophy into words of one syllable, and the Joads translate it into action.» (F. I. Carpenter, «The philosophical joads», dans J. Steinbeck, ouvr. cité, p. 709.)

31 L'intérêt de Steinbeck pour les études naturalistes véritables, et notamment pour la biologie marine (tel qu'il le développe notamment dans son ouvrage The Sea of Cortes), ne fait pas de lui pour autant un simple «peintre animalier» de la vie humaine. Sa vision «organique» est plus complexe, et principalement parce qu'elle tient compte de l'univers technique et machiniste dans lequel évolue la société américaine du XXe siècle. Voir à ce sujet, entre autres, R. J. Griffin et W. A. Freedman, «Machines and animals: Pervasive motives in The Grapes of Wrath», dans J. Steinbeck, ouvr. cité, p. 769-783. Mais il faut voir, en dépit de ce qu'en disent ces deux auteurs, que le mariage entre l'univers technique et la vision organique de Steinbeck s'établit sur un plan fondamental: c'est dans la communication, et notamment dans la définition d'une communication pragmatiste dont on trouve le modèle dans les médias, un modèle largement exploité par toute l'école de Chicago, que ce mariage entre technologie et vie organique trouve son assise fondamentale. Nous reviendrons sur cette question dans la prochaine section. 
qui les unit immédiatement dans leur action ${ }^{32}$. La signification subjective de l'action individuelle ne trouve de résonance que dans cet horizon social immédiat de l'action commune ${ }^{33}$. Cette action ne peut donc jamais être comprise autrement que comme «action sociale», et ne peut jamais renvoyer à autre chose qu'à une situation empirique de vie qui accomplit sa signification dans cette même action sociale.

Sur ce plan, il peut donc très certainement y avoir confusion entre sociologie, littérature et travail social, puisque chaque domaine peut être ramené en fait, comme tout autre domaine et même comme la vie quotidienne dans le «monde vécu», à sa simple dimension d'«action sociale». Et parce que c'est l' «efficace» empirique de cette action qui devient l'aune à partir de laquelle sa propre signification se mesure ou même, en suivant Blumer, que sa validité s'établit - on comprend aisément qu'une certaine confusion des domaines et des genres devienne tout à fait possible. Dès lors, la fonction symbolique propre à l'activité scientifique, fonction qu'on retrouverait essentiellement investie dans la forme typique d'objectivation que constitue le concept, perd complètement sa visée d'universalité référentielle, pour devenir en quelque sorte un agent de transformation sociale, à consonance nécessairement particulière. Le concept qui détermine la spécificité de sa fonction symbolique dans sa capacité à saisir la réalité de l'ordre général du monde s'épuise ainsi dans sa réalisation «immédiate». Et pour cette raison même, peut-être le concept scientifique ne peut-il jamais être le représentant le plus fidèle d'une efficacité qui se comprend réellement comme expression immédiate de la réalité singulière; car le «monde vécu» semblerait bien pouvoir se suffire en fait à lui-même dans cette tâche. Or dans ce domaine cependant, c'est l'art qui est le médium tout désigné pour élever symboliquement la particularité de l'expérience singulière à l'universalité. On voit ici, et peut-être très exactement, comment et pourquoi, lorsque la sociologie est rendue à ce point, il peut devenir tentant pour elle de «faire de la littérature», afin de regagner en universalité ce qu'elle a perdu dans son effort méthodologique de rapprochement d'une réalité singulière, en

32 Cette vision de Steinbeck ressort particulièrement dans deux de ses romans les plus «engagés» que sont The Grapes of Wrath et In Dubious Battle. Le Steinbeck de Tortilla Flat ou de Of Mice and Men a adopté une position légèrement différente - et d'ailleurs peut-être plus intéressante sur le plan proprement esthétique.

33 Le modèle théorique le plus complet développé à cet égard sera évidemment celui de Talcott Parsons, qui publie The Structure of Social Action en 1937. Le «systémisme» qui est en germe dans le fonctionnalisme de Parsons repose, lui aussi, sur une vision naturaliste, et l'on pourrait montrer certaines convergences qui se déploient, envers et contre toute apparence et malgré les oppositions parfois féroces, entre le pragmatisme de l'école de Chicago et le fonctionnalisme. 
abandonnant sa visée proprement conceptuelle. Dans le cas de Steinbeck, il semble en tout cas que c'est par l'universalité de la signification exprimée à travers la situation particulière des migrant workers que les acteurs du contexte de la société américaine du temps du New Deal avaient compris son roman, et que la revue Social Work Today avait jugé de son apport significatif - sinon universel - dans l'ordre des «transformations sociales» requises par la situation du temps. Et pourtant cette confusion, dans le contexte de l'époque, n'était pas inévitable. Nous venons de voir à quoi elle tenait; il importe de voir également comment nous pouvons au moins aujourd'hui éviter de la reproduire. Car en fait l' «efficace» tant recherchée dans ce cadre est celle de la communication, définie de façon essentiellement pragmatiste.

\section{Sociologie et société: culture et sciences de la culture à l'âge de la communication pragmatiste}

Ce que, à mon sens, l'on peut dire de plus général du contexte sociétal qui voyait science et littérature (et même travail social, journalisme de reportage et activisme politique) converger dans leurs aspirations respectives, particulièrement pendant la période du New Deal, est que la fonction symbolique qui animait alors chacun de ces domaines devait répondre d'une efficace pratique. C'est-à-dire d'une efficacité éprouvée dans l'action sur le monde, dans l'action immédiate comprise comme action sociale, afin que la fonction symbolique assumée à cette occasion remplisse à la fois son exigence d'expression correspondant réellement à la vie du «groupe social», à ses désirs et à sa volonté de transformation de la réalité qui s'incarnent autant dans l'action (sociale ou politique) que dans la perception, la représentation ou la conceptualisation de la réalité. Cette fonction symbolique générale est celle que le pragmatisme a appelé commmunication ${ }^{34}$. C'est en effet la communication pragmatiste, définie dans une perspective naturaliste par ses dimensions sensibles et spirituelles, structurée par son orientation symbolique fonctionnelle correspondant à l'expression de la «nature» même du groupe social et de la société, qui apparaissait comme la synthèse par laquelle s'opérait une redéfinition de la réalité capable d'unifier le monde vécu et son «système» (ou son organisation

34 Voir J. Dewey, art. cité, 1981, p. 132-190; G. H. Mead, Mind, Self, Society, édition préparée par C. Morris, Chicago, University of Chicago Press, 1963 (1934), p. 253 260; R. Park, «Reflections on communication and culture», The Crowd and the Public and Other Essays, Chicago, University of Chicago Press, 1972, p. 98-116; C. W. Morris, Six Theories of Mind, Chicago, University of Chicago Press, 1932, p. 274-330. 
d'ensemble ${ }^{35}$ ). Le rapport entre sciences sociales et société est donc défini par cette exigence de communication pragmatiste qui s'incarne notamment dans la sociologie de l'école de Chicago ${ }^{36}$ - comme dans le réalisme social de Steinbeck.

On peut s'interroger à cet égard sur la manière dont la communication pragmatiste apparaît finalement se poser expressément comme une norme dans la société américaine; en effet, tant du point de vue de la littérature que du point de vue de la sociologie, ou de ce à quoi nous renvoie aujourd'hui cet épisode culturel de la rencontre historique entre le réalisme social et l'école de Chicago dans la communication pragmatiste, nous pouvons tenter de saisir la suite des enjeux débattus à cette occasion. C'est donc par l'examen rapide de ces trois aspects de la question que je conclurai ici.

Le réalisme social qui imprègne l'esthétique de Steinbeck, particulièrement dans son roman The Grapes of Wrath, ne constitue certes pas la seule issue pour la conscience littéraire américaine du temps. En fait, c'est dans les modalités différenciées de participation à l'ordre symbolique plus général de la communication qui s'imposait alors comme réalité objective que les expressions littéraires diverses trouvaient leur propre spécificité; en adoptant dans certains cas une attitude critique à l'endroit du phénomène des médias ou de la communication pragmatiste, la conscience littéraire de l'époque ménageait un espace pour une compréhension dialectique de

35 Contrairement à l'idée défendue encore tout récemment par E. Rochberg-Halton («Jürgen Habermas's theory of communicative etherealization», art. cité, et Meaning and Modernity. Social Theory in the Pragmatic Attitude, Chicago, University of Chicago Press, 1986), le rapport entre l'analyse pragmatiste et l'analyse fonctionnaliste et systémiste ne tient pas à l'opposition entre une expression «naturelle» de la vie sociale et un excès de formalisation «rationaliste», mais il apparaît davantage dans le rapprochement entre les deux démarches sur le plan ontoépistémologique, que les modalités analytiques («empiriques» d'un côté, «formalisées» de l'autre) se chargent de distinguer. Ainsi, on peut très bien percevoir la jonction explicite entre le pragmatisme et le fonctionnalisme chez un Robert Park, dans sa théorisation précoce des «mouvements sociaux» et du procès d'institutionnalisation auquel ils peuvent donner lieu. Voir R. Park, «Symbiosis and socialization: A frame of reference for the study of society», dans R. Park, ouvr. cité, p. 117-142.

36 Sur cette question, je me permets de renvoyer à ma thèse de doctorat: J.-F. Côté, Tradition, modernité, postmodernité dans la société américaine: contribution à l'étude critique du concept pragmatiste de communication des sciences sociales américaines dans une problématique de la socialité contemporaine, Toronto, York University, 1992, $531 \mathrm{p}$. 
communication ${ }^{37}$. On peut alors s'interroger sur la position de Steinbeck à cet égard pour évaluer la part finalement assez faible de son apport critique au regard de cette réalité; dans la mesure où son expression littéraire se rapproche du «reportage», Steinbeck fait en quelque sorte le jeu de l' «effacement de la distance» par rapport à la vie sociale «organique» de la société américaine de l'époque - ce qui contribue également à l'effacement de la position dialectique de la conscience de soi de l'écrivain ${ }^{38}$. On peut ainsi se demander quel type de littérature continue, aujourd'hui, d'entretenir cet espace critique face à la communication pragmatiste, pour montrer comment la réflexion esthétique se poursuit à travers la problématisation du phénomène de communication lui-même ${ }^{39}$.

La science de son côté, et particulièrement la sociologie, donnait entre autres par les analyses de l'école de Chicago la mesure de sa compréhension du phénomène de la communication pragmatiste dans la pleine positivité qu'elle lui accordait. Ici, ce à quoi nous sommes renvoyés, c'est à l'évaluation des deux positions épistémologiques évoquées plus haut: celle des sciences de la nature qui tablent sur un «effacement de la distance» par rapport à leur objet -- en omettant toutefois la reconnaissance de leur rôle actif dans la production de la connaissance à son endroit -; et celle des sciences de la culture, qui tablent de leur côté à l'inverse sur la reconnaissance d'une «production de la distance» comme modalité spécifique de participation à la compréhension de la réalité sociale, distance qui est avant tout celle d'un espace symbolique de médiation. C'est à mon avis dans cette orientation des sciences de la culture que la simple «positivité» du phénomène de communication pragmatiste, dans sa définition naturaliste, peut être évitée. La démarche réflexive des sciences de la culture, qui reconnaît son rapport dialectique avec son objet, apparaît ainsi comme une possibilité de dépassement de la perception et de la conception de la communication pragmatistes dans ses termes naturalistes - et cela, déjà dans la façon dont on fait de cette

37 Sur cette question, je renvoie à mon article, J.-F. Côté, «Dialectique de la communication dans le roman américain», Discours social/ Social Discourse, vol. 7, nos 3-4, été-automne 1995, p. 113-126.

38 Et comme l'écrivait R. Park, dans ce contexte de la communication pragmatiste, «[...] l'obstacle ultime à la communication, c'est la conscience de soi» (R. Park, «La communauté urbaine. Un modèle spatial et un ordre moral», dans L'école de Chicago. Naissance de l'écologie urbaine, textes traduits et présentés par Y. Grafmeyer et I. Joseph, Paris, Éditions du Champ Urbain, 1979, p. 193-207.

39 Les propositions néopragmatistes de E. Rochberg-Halton (Meaning and Modernity: Social Theory in the Pragmatic Attitude, Chicago, University of Chicago Press, 1986, p. 230-277) concernant des rapprochements actuels entre l'entreprise sociologique et le réalisme fantastique peuvent toutefois laisser songeur... 
conceptualisation et de ces développements théoriques et pratiques des productions culturelles, sociales et historiques. Est-ce à dire pour autant que cette position des sciences de la culture renonce à s'ouvrir à la «sensibilité» du monde social, à ce qu'exprime par exemple réellement le «monde vécu», pour se réfugier dans l'abstraction froide d'une analyse «rationaliste» qui ne vise que la définition conceptuelle (sinon systémique) des choses?

Cette position qui tient à opposer de manière définitive la sensibilité et la conceptualisation est, comme je l'ai mentionné plus haut, une position intenable sur le plan proprement phénoménologique. La sensibilité fait partie d'une démarche qui reconnaît que c'est l'existence humaine, dans ses manifestations phénoménales (et donc «sensibles»), qui constitue son objet propre. Mais elle reconnaît également que cette existence phénoménale est constituée, dans le cas de la réalité humaine, par sa dimension symbolique - exprimée autant sur le plan gestuel que sur le plan linguistique - et que cette dimension, en tant qu'elle peut être comprise, se déploie dans un espace dialogal qui représente une modalité fondamentale de l'expérience humaine ontologiquement structurée par la dialectique ${ }^{40}$. Il appartient donc à la compréhension sociologique de saisir que la logique de l'expression phénoménale représente en fait toujours une logique dialectique, et que c'est dans ce procès qu'elle-même intervient. C'est cet espace dialectique qui est le milieu propre où se déploie son propre discours, qui fait nécessairement apparaître une certaine «logique» des choses, ne serait-ce que sur le plan de la compréhension qu'il en donne. On peut très bien concevoir alors que des «niveaux» de formalisation tiennent à respecter la subjectivité qui s'exprime (par le biais d'une «sociologie des émotions $\left.{ }^{41} »\right)$, ou même la particularité singulière d'une simple individualité (par le biais biographique des «histoires de $v^{4 e^{42}}$ ), ou encore la «subjectivité sociale organisée» (par le biais des analyses de

\footnotetext{
40 On peut aller plus loin et mentionner que cet espace dialectique devient, dans le cadre de la théorisation sociologique, le lieu d'une distance spatio-temporelle qui exige la reconnaissance de l'histoire ou de l'historicité des phénomènes. Sur cette question, je renvoie à mon article, J.-F. Côté, «Critique de la synchronicité de la phénoménologie pragmatiste: la sociologie entre communication et historicisation», dans D. Arsenault et F. Piron (dir.), La construction sociale du temps, Québec, Septentrion, à paraître.

41 Comme le suggèrent entre autres J.-F. Laé et N. Murard, ouvr. cité, ou N. Denzin, art. cit., ou même E. Rochberg-Halton, «Jürgen Habermas theory of communicative etherealization», art. cité.

42 Comme le proposait déjà Wilhelm Dilthey (Le monde de l'esprit, t. 1, trad. M. Rémy, Paris, Aubier, 1947, p. 145-317) à la fin du XIXe siècle, dans l'optique du développement des sciences de l'esprit ou des sciences de la culture.
} 
mouvements sociaux ${ }^{43}$ ), mais dans chacun de ces cas, il reste que l'exigence minimale du discours sociologique apparaît dans sa capacité à faire sortir, à «abstraire», ces expressions de leur simple autoréférentialité pour les faire participer d'une compréhension plus large ou plus systématique. Dans ce sens, la logique conceptuelle n'est pas détachée de toute sensibilité esthétique; au contraire, son propre projet de compréhension est même entièrement déterminé par elle - et cela jusque dans le plaisir de la compréhension. C'est à mon sens ce qu'il importe de reconnaître comme étant le propre de l'activité de connaissance, et c'est entre autres la tâche des sciences de la culture de rendre explicite cette position réflexive qui est la leur. En cela, les sciences de la culture ne sont aucunement en déficit de quoi que ce soit par rapport aux sciences de la nature; seulement, elles sont très certainement différentes dans leur projet, puisqu'elles ont toujours notamment pour tâche de faire reconnaître la présence humaine dans l'entreprise de connaissance de la réalité, et cela autant pour la réalité humaine que pour la réalité naturelle.

Jean-François CÔTÉ

Département de sociologie

Université du Québec à Montréal

Résumé

Dans le contexte américain des années trente, la rencontre entre la littérature et la sociologie peut être en partie évaluée dans une comparaison entre le réalisme social de John Steinbeck, exemplifié dans son roman The Grapes of Wrath (Les raisins de la colère) et les développements méthodologiques de l'interactionnisme symbolique de Herbert Blumer, qui veulent assurer une suite à la tradition des études empiriques de l'école de Chicago. Cette rencontre, qui pose une possibilité de saisir l'expression sociale dans son contexte «naturel» et qui met en œuvre une méthodologie «naturaliste» en sociologie faisant appel plus largement à des concepts hérités de la philosophie du pragmatisme, est en fait portée par l'idée d'une possibilité de communication «immédiate» de la vie sociale. On peut prendre la mesure critique de ce qui s'exprime alors dans ce contexte, en fonction des exigences de la réflexion actuelle en sociologie concernant les modalités d'analyse et d'expression du «monde vécu», ainsi que des liens possibles à établir dans cette perspective entre littérature et sociologie.

43 Comme veulent le faire la sociologie du «retour de l'acteur» ou la sociologie des mouvements sociaux mentionnés par J. Hamel, (art. cité). 
Mots-clés: littérature, sociologie, États-Unis, communication, pragmatisme, Herbert Blumer, interactionnisme symbolique, John Steinbeck, réalisme social.

\section{Summary}

In the United States of the 1930s the meeting between literature and sociology can be partly evaluated by a comparison of John Steinbeck's social realism, exemplified in his novel The Grapes of Wrath, with the methodological developments of Herbert Blumer's symbolic interactionism, which was intended as a continuation of the Chicago School's empirical studies. This meeting, which provides the possibility of studying social expression in its "natural" context and deploys a "naturalist" methodology in sociological analysis relying largely on concepts inherited from pragmatism, is in fact infused by the idea of the possibility of "immediate" communication of social life. One can critically examine what was expressed in that context within the critical framework of current sociological analysis pertaining to the modalities of analysis and expression of the "lived world" as well as the possible links to be established in this perspective between literature and sociology.

Key-words: literature, sociology, United States, communication, pragmatism, Herbert Blumer, symbolic interactionism, John Steinbeck, social realism.

\section{Resumen}

En el contexto norteamericano de los años treinta, se puede evaluar en parte el encuentro entre literatura y sociología mediante una comparación del realismo social de John Steinbeck, ejemplificado por su novela The Grapes of Wrath (Las Uvas de la Ira) con los desarrollos metodológicos del interaccionismo simbólico de Herbert Blumer, cuya intención es asegurar la continuación de la tradición de estudios empíricos de la escuela de Chicago. Este encuentro, que propone la posibilidad de captar la expresión social en su contexto «natural» y que implementa en sociología una metodología «naturalista» haciendo amplio uso de conceptos heredados de la filosofía del pragmatismo, es vehiculado de hecho por la idea de la posibilidad de una comunicación «inmediata» de la vida social. Se puede así apreciar críticamente lo que se expresa en aquel contexto, en función de las exigencias de la reflexión sociológica actual en lo que respecta a las modalidades de análisis y de expresión del «mundo de la vida»; siendo posible también, 
desde esta perspectiva, interrogarse acerca de las relaciones que puedan establecerse entre literatura y sociología.

Palabras claves: literatura, sociología, Estados Unidos, comunicación, pragmatismo, Herbert Blumer, interaccionismo simbólico, John Steinbeck, realismo social. 